Meta

Journal des traducteurs

Translators' Journal

\title{
Théorie et pratique de la traduction professionnelle : différences de point de vue et enrichissement mutuel
}

\section{Jean Darbelnet}

Volume 25, numéro 4, décembre 1980

URI : https://id.erudit.org/iderudit/002479ar

DOI : https://doi.org/10.7202/002479ar

Aller au sommaire du numéro

Éditeur(s)

Les Presses de l'Université de Montréal

ISSN

0026-0452 (imprimé)

1492-1421 (numérique)

Découvrir la revue

Citer cet article

Darbelnet, J. (1980). Théorie et pratique de la traduction professionnelle : différences de point de vue et enrichissement mutuel. Meta, 25(4), 393-400. https://doi.org/10.7202/002479ar d'utilisation que vous pouvez consulter en ligne. 


\section{Théorie et pratique de la traduction professionnelle: différences de point de vue et enrichissement mutuel}

JEAN DARBELNET

Tel qu'il a été proposé et accepté, le titre de ma communication d'aujourd'hui commence par les mots théorie et pratique. Étant donné que je vais dans un instant m'inscrire en faux contre la prédilection de notre époque pour tout ce qui est théorie et méthode, c'est-à-dire pour la forme préférée au fond, ou, si l'on préfère, à la substance, je voudrais d'abord expliquer clairement que cette exclusive que je semble lancer contre la théorisation n'exclut nullement dans mon esprit la nécessité de la réflexion. À côté des praticiens férus de théorie, devenus assez nombreux de nos jours, il y a sans doute encore, en particulier dans le domaine de la traduction littéraire, des traducteurs intuitifs qui suivent leur instinct et font fi de la théorie, ce qui ne nuit pas nécessairement à leur succès. Mais, comme je viens de le noter en passant, cette façon de procéder est surtout compatible avec un certain type de traduction. Au Canada, c'est la traduction technique qui donne le ton et qui, de par sa nature, impose certaines exigences. Quoi qu'il en soit, je me garderai bien de nier non seulement l'utilité, mais la nécessité de réfléchir à ce qu'on fait, de savoir où l'on va quand on entreprend une tâche et comment on en vient à bout. Je pense au coupe-papier de Sartre : l'artisan qui l'a fait l'a vu à l'état de projet, en a conçu les caractéristiques avant d'en travailler la matière. Et celui qui traduit, qu'il le veuille ou non, part d'une certaine conception qu'il se fait de la traduction. Mon objection ne vise pas cette activité pensante, à la fois naturelle et désirable. $\mathrm{Ce}$ que je reproche à la théorie, c'est de proliférer subjectivement, chacun ayant le droit d'échafauder la sienne. Elle est au fond une expression d'individualisme, une forme de créativité. Les théories à l'éclosion desquelles nous assistons, se juxtaposent, plutôt qu'elles ne s'appuient les unes sur les autres; elles ne sont pas cumulatives comme les découvertes des sciences physiques et naturelles. Disons-le, une théorie n'est pas une découverte. Au fond, mon point de vue sur ce point n'est pas très différent de celui de Valéry sur la philosophie en général. Je soupçonne en outre que si la théorisation jouit d'une telle faveur, c'est en partie parce qu'elle est sécurisante. Comme telle, elle a l'inconvénient de faire oublier ou négliger d'autres facteurs d'une bonne traduction, en particulier une connaissance aussi approfondie que possible des deux langues en présence. Je ne suis pas censé parler aujourd'hui d'enseignement. Je me permettrai seulement une simple remarque pour clore cette partie de mon exposé. En tant qu'enseignant je crois indispensable de faire réfléchir les étu- 
diants de traduction à ce qu'ils font quand ils traduisent. Je suis beaucoup moins sûr qu'il faille institutionnaliser cette réflexion en la systématisant dans le cadre d'un cours sur la théorie de la traduction, cours qui ne devrait apparaître à mon avis qu'au second cycle.

À la théorie je préfère donc les principes, dont le maniement me paraît plus facile et plus sûr. D'abord on peut les isoler, les concrétiser. On peut aussi les rejeter ou les adopter séparément avec la même aisance. Au hasard d'une lecture, j'ai remarqué que le maréchal Foch, qui compte parmi les théoriciens de la guerre moderne, a intitulé l'un des deux ouvrages qui consacrent sa réputation sur ce pont: Des principes de la guerre, dont il disait, lui aussi, qu'elle était un art simple, tout d'exécution. Eh bien, justement, je trouve qu'au fond la traduction, à la fois art et science, est quelque chose d'essentiellement simple, puisqu'il ne s'agit après tout que de transvaser le contenu d'un récipient dans un autre, et que c'est l'exécution de cette opération (ne rien renverser) qui compte.

Les principes ne sont pas sujets à des variations individuelles. On les accepte ou on les rejette. Mais ce faisant, l'individu marque néanmoins son sentiment personnel, donc subjectif. Donnons un ou deux exemples. C'est un principe aujourd'hui généralement admis qu'il ne faut pas traduire un texte un mot à la fois, sans tenir compte des unités de sens qu'il leur arrive de former en s'associant. C'est un autre principe, discutable cette fois, que le sens prime la forme. Mais si je l'accepte - et tout le monde ne sera pas d'accord - je dois accepter les conséquences de cette option, qui, comme les autres possibles, comporte inévitablement des inconvénients. Il y a forcément un jugement personnel dans l'adoption ou le rejet d'un principe. Il en va de même de celui-ci qui est fondamental dans l'optique où je me place et que j'ai effleuré tout à l'heure. Pour être bon traducteur, il faut avant tout connaître les deux langues aussi bien que possible. Cela va sans dire, m'objectera-t-on. Oui, mais on tend à l'oublier, et cela va mieux en le rappelant.

Mais il y a plusieurs façons de connaître une langue et il convient de noter à cet égard une distinction entre la langue de départ (en principe la deuxième langue du traducteur) et la langue d'arrivée (en principe sa langue maternelle). Il doit connaître la première dans ses nuances les plus fines et posséder autant que faire se peut les ressources de la seconde. Les expressions «connaitre les nuances», "posséder les ressources» s'appliquent respectivement à une connaissance passive et à une connaissance active. On remarquera que dans ce dernier cas nous évitons de dire «à fond». Personne ne connaît une langue à fond, pas même la sienne.

Cette distinction entre connaissance, au sens de compréhension, et possession, au sens d'utilisation active, vaut également, mutatis mutandis, pour cet autre important secteur de l'opération traduisante qu'est l'interprétation. On ne peut pas dire que le passage de la consécutive à la simultanée ait vraiment modifié notre conception de l'opération traduisante dans ce secteur. On notera cependant qu'en étant plus rapide, la simultanée a accentué l'instantanéisme de l'interprétation. Elle fait appel à une appréhension immédiate du sens de sorte 
que l'interprète s'arrête moins que jamais à ce que l'ESIT, dans sa doctrine de l'interprétation, appelle les significations linguistiques, pour capter globalement le message de l'intervenant.

Dans le domaine de la traduction écrite, la première en date des spécialités qui se sont constituées récemment est sans doute la terminologie, qui d'ailleurs, en tant que discipline, existe en dehors de la traduction. Qui dit terminologue ne dit pas nécessairement traducteur, et vice versa. En principe le terminologue fait de la recherche dite terminologique, qui lui permet à l'occasion de venir en aide au traducteur. Mais l'abondance des textes techniques à traduire, en particulier dans un pays officiellement bilingue comme le Canada, ne pouvait manquer d'amener les traducteurs à s'initier à la terminologie. La STQ a maintenant une section de terminologie. L'importance que cette discipline a prise au Québec tient à ce qu'elle est devenue assez brusquement une nécessité lorsqu'on a proclamé le français langue de travail, ce qui a obligé les travailleurs, dans de nombreux cas, à acquérir le vocabulaire spécialisé dont ils avaient besoin pour exercer leur profession dans leur langue maternelle. La nouveauté de la chose a naturellement engendré un certain engouement qui confine parfois à la mythification. Certains sont allés jusqu'à assigner à la terminologie un domaine entièrement à part, à lui conférer une complète autonomie. On ne saurait trop s'élever contre cette prétention qui ne correspond pas à la réalité. Il est exact que la lexicologie et la terminologie ne traitent pas leurs unités de la même façon, le mot de la langue générale et le terme technique n'ayant pas des comportements identiques. Mais il ne faut pas oublier que les termes techniques sont dans la langue, que leur création, le cas échéant, utilise les formants (racines et affixes) de la langue et que le discours terminologique ne peut fonctionner qu'à l'aide de la syntaxe qu'on apprend à l'école. Nous disposons de deux expressions pour marquer la distinction entre le général et le technique: vocabulaires spécialisés, langues de spécialités. La première ne porte à aucune confusion; on sait où un vocabulaire spécialisé commence et où il finit. Il n'y a aucun inconvénient à parler de langues de spécialités à condition de ne pas perdre de vue que langue, dans ce sens, ne désigne pas une entité délimitable comme quand il s'agit d'une langue nationale. Ce sens de langue est d'ailleurs identifié dans les dictionnaires (langue philosophique, langue du Palais). Le continu qui existe entre lexicologie et terminologie apparaît aussi dans le fait qu'il y a deux sortes de termes techniques, technique par la forme et par le sens (sidérurgie, radiographie) et technique par le sens seulement (ex.: to recognize, au sens de donner la parole à). La distinction est en grande partie une question de transparence. On sait que l'esprit humain se laisse facilement guider par les apparences. En tant que profanes, nous reconnaissons comme technique tout mot dont l'aspect insolite nous surprend. C'est une réaction de non-initié. Par contre nous ne remarquons pas aussi vite le sens technique d'un mot usuel, qui nous est familier, mais pas nécessairement dans ce sens-là. Et même là il y a des degrés. Dans un premier temps je me dis que dans «arroser la viande» arroser, mot usuel, est ici pris dans un sens technique, sans doute parce que la cuisson de la viande à 
la broche ne fait pas partie de ma vie quotidienne. Poussant plus loin ma réflexion, je découvre qu'en fait arroser est aussi un terme technique par le sens quand il s'agit des fleurs du jardin, mais le sens technique s'efface derrière la banalité apparente de l'opération. Je dis «apparente», car l'arrosage des fleurs est une opération technique qui demande certaines précautions et dont les manuels d'horticulture font sans doute état. En fait, est technique tout terme pris au sens propre et étroitement lié à un domaine d'emploi. Il est donc très difficile de dire où commence et où finit le vocabulaire spécialisé, et nous ne savons jamais quand un mot du vocabulaire courant va s'annexer un sens technique. Accueil est devenu technique il n'y a pas très longtemps dans le vocabulaire des voyages. Et naturellement il ne se traduit alors ni par reception ni par welcome, pas plus que le recognize, cité plus haut, ne se rend par reconnaître.

Il peut paraître excessif de classer la révision parmi les nouveaux secteurs auxquels la diversification de la profession de traducteur a donné naissance. $\mathrm{Ce}$ n'est pas à proprement parler une nouvelle invention. Mais comme d'autres activités de notre époque, elle s'est institutionnalisée, et c'est ce qui lui a donné une nouvelle figure. L'accroissement des effectifs de traducteurs d'expérience limitée dans les deux fonctions publiques et dans l'entreprise privée a rendu nécessaire un contrôle généralisé de la qualité de leur travail. Il a même fallu songer à initier les étudiants, au cours de leur scolarité, à cette spécialité qui pouvait leur échoir assez tôt dans leur carrière. Dans une communication intitulée "Traduction ou rédaction», faite en 1977, M. Horguelin nous informe que la révision s'enseigne à l'Université de Montréal depuis 1974. C'est une activité plus simple que l'interprétation ou la recherche terminologique, puisqu'il s'agit seulement d'exercer un savoir déjà acquis. Mais elle nous concerne ici dans la mesure où elle permet de réfléchir aux conditions dans lesquelles se déroule le processus de la traduction et à la justification de ce contrôle. Je me permettrai donc d'exposer brièvement ma propre réflexion à ce sujet.

Une première considération porte sur la difficulté de la traduction et, par voie de conséquence, de la révision. On ne se rend pas toujours compte que la tâche du traducteur est plus difficile, à certains égards, que celle de l'écrivain, que l'on place cependant au-dessus. Non pas que la création, qui est le propre de celui-ci, soit chose facile, mais du moins écrit-il librement parce qu'il veut écrire, parce qu'il a quelque chose à dire. Il choisit lui-même ce qu'il va dire et il jouit pour le dire d'une certaine latitude dans le choix de ses moyens d'expression. Cette liberté du fond et dans une certaine mesure de la forme est refusée au traducteur. Cela est évident dans la traduction littéraire subordonnée à une création artistique. Ce l'est aussi dans la traduction générale ou technique.

Un deuxième point concerne plus spécialement le côté administratif de la révision, mais dérive de ce qui précède. On est porté à croire qu'il suffit de prendre un bon traducteur pour en faire un réviseur, et, à première vue, cela devrait devoir régler la question. En fait, le problème n'est pas tout à fait aussi simple et je voudrais faire intervenir ici deux notions que je juge perti- 
nentes : celle d'idiolecte et celle de pluralisme de l'usage. Elles sont d'ailleurs connexes et valent aussi pour la situation du professeur corrigeant les copies de ses étudiants, sauf que dans ce dernier cas les options de l'étudiant lui sont dictées, au moins en partie, par le cours qu'il suit, alors que le cas du traducteur révisé comporte un plus large choix. C'est là le hic. Le réviseur manie sa langue avec sûreté et c'est pour cela qu'il a été choisi. Mais son usage n'est pas le seul possible dans les limites de ce qui est permis. Chacun a sa façon d'employer sa propre langue; chacun a son idiolecte. Le pluralisme de l'usage, de l'usage accepté, recouvre un certain nombre de variantes, ou d'idiolectes sur lesquels il arrive que le réviseur soit tenté de compter fautif un emploi qui ne l'est pas, qui est simplement différent du sien, qui relève d'un autre idiolecte dans les limites du bon usage. On raconte que Winston Churchill révisé par un lecteur de son éditeur, qui avait fait sauter une préposition en fin de phrase, rétablit sa rédaction originale et l'annota comme suit : «This, my dear man, is the sort of thing up with which I will not put.» En français, certains réviseurs accepteront réaliser au sens de se rendre compte, d'autres le rejetteront. D'autres encore considèrent que activités (au pluriel) est un anglicisme, alors qu'il est facile de démontrer que tel n'est pas le cas. Tout cela pour dire que la fonction de réviseur demande un bon idiolecte et une bonne connaissance du bon usage actuel avec les variantes qu'il comporte inévitablement. Le moyen de réaliser cette condition est relativement simple. Il faut avoir observé l'usage et y avoir réfléchi. Pour cela deux moyens sont indispensables; la motivation et le temps. Les réviseurs sont souvent trop jeunes pour les tâches qu'on leur confie.

Si curieux que cela paraisse, la rédaction est bel et bien devenue, au moins pour quelque temps, une spécialité de la traduction. La communication de M. Horguelin devant la STQ en décembre 1977 est consacrée à la place qu'était en train de prendre la rédaction dans la profession de traducteur au Canada. Et à l'automne de la même année l'université de Sherbrooke avait institué un programme d'enseignement destiné à former des rédacteurs. Mais comme le rappelle $\mathbf{M}$. Horguelin, il est question de rédaction dans Meta dès mars 75 et en mars 76 le Bureau des traductions songe à organiser cette activité pour ses propres besoins.

Nous disions à l'instant que ce phénomène nous apparaissait comme curieux. C'est là une réaction spontanée et que la réflexion devrait corriger. A première vue, il paraît en effet surprenant que soit enseigné comme spécialité ce que l'on est censé apprendre à l'école. Mais sur le continent nordaméricain il est admis depuis longtemps que l'on apprend à tout âge, et d'autre part, dans l'enseignement du français, l'école insiste moins sur l'écrit que naguère. En outre le problème de la rédaction, puisque problème il y a, n'est pas aussi simple qu'il y parait de prime abord.

De ce qui se dit ou s'écrit sur la rédaction depuis 1975 , se dégagent deux conceptions. La rédaction parallèle (ce terme semble préférable à celui de bilingue, qui a aussi été employé) s'oppose à la rédaction dite unilingue, non sans une certaine confusion qu'il serait peut-être bon de dissiper. 
La première réflexion qui se présente à l'esprit quand on prend connaissance de cette nouvelle préoccupation est qu'on ne peut pas bien traduire sans rédiger. L'effort rédactionnel est nul, de toute évidence, si l'on traduit mot à mot; il peut être considérable si l'on sait se détacher des mots de l'original et si pour rendre le sens qu'ils véhiculent, on a recours dans la langue d'arrivée à une forme non seulement correcte mais idiomatique et conforme à la démarche naturelle de la langue quand elle est livrée à sa propre impulsion.

Mais dans un second temps, on constate que la question peut se présenter sous un aspect assez différent. Il y aurait alors non pas traduction, mais rédaction parallèle à celle qui a donné naissance à l'original. On pense à Freud qui, pour occuper des loisirs forcés, s'était mis à traduire de l'anglais en allemand en lisant une page de l'original, puis repoussant le livre, en mettant par écrit ce qu'il avait retenu de sa lecture. C'est un exercice intelligent si on sait s'en acquitter correctement et qui demande à être fait par des traducteurs supérieurement entraînés, capables de drainer tous les éléments de sens de l'original et de les repenser dans la langue d'arrivée. Une telle approche marquerait un progrès dans le domaine de la traduction, dont elle continuerait à relever puisque le point de départ est un texte conçu et rédigé dans une autre langue que le français. Mais outre sa difficulté cette façon de procéder serait onéreuse au point de vue temps. N'oublions pas que des diverses façons de traduire, la plus rapide est le mot à mot.

Si on regarde la situation dans son ensemble et à ce stade de son évolution, on a l'impression que dans ce qui précède la part ainsi réservée à la rédaction dans la traduction pourrait bien n'être qu'une demi-mesure destinée à être dépassée, en cédant de plus en plus de terrain à la rédaction unilingue, dont la matière traitée ne doit rien à l'anglais, puisqu'elle a été conçue uniquement en français.

La faveur dont jouit depuis quelque temps la traduction unilingue au Québec se rattache à un phénomène psychologique d'ordre collectif qui pousse les Québécois à réaffirmer leur identité culturelle en voulant produire de plus en plus des textes français conçus en français et non dérivés, d'une façon ou d'une autre, de l'anglais. Il est difficile de ne pas y voir l'impact des lois 22 et 101. Pierre Daviault disait que les Canadiens français étaient un peuple de traducteurs en ce sens que la plupart des traducteurs au Canada étaient des francophones (ou traduisaient alors surtout vers le français) et que dans ce que lisait le public canadien-français, les traductions de l'anglais formaient l'élément principal. Il semble bien que maintenant les Québécois veuillent se libérer de ce qui leur apparaît comme un servage et lire des textes non seulement rédigés mais aussi conçus dans leur langue. La conséquence de cette nouvelle orientation est que le volume des traductions vers le français a diminué alors qu'augmente celui des traductions vers l'anglais au fur et à mesure que l'administration québécoise entreprend de mettre à la disposition du public anglophone, québécois ou d'outre-frontières, les textes qui, bien que le concernant, ont d'abord été élaborés en français. 
On remarque que dans l'entreprise, les services qui s'occupent de traduction s'appellent souvent services linguistiques. Cette désignation tient compte du fait qu'ils ne font pas que de la traduction. Il paraît d'ailleurs assez indiqué de confier la rédaction, au moins en partie, à des traducteurs qui de par leur formation devraient être en mesure d'assurer ce service. De toute façon il ne faut pas s'attendre, à Québec ou ailleurs, qu'on puisse se passer de la traduction dans le monde moderne. On peut donc prévoir que le personnel des services linguistiques comprendra de plus en plus des rédacteurs unilingues aussi bien que des traducteurs. La cohabitation de ces deux catégories de spécialistes devrait d'ailleurs être bénéfique pour la traduction.

Nous en venons maintenant à une question d'un autre ordre. Il ne s'agit plus de la diversification de l'opération traduisante, mais d'une notion qui, elle aussi, est relativement récente et peut s'appliquer aux secteurs que nous venons de passer en revue. C'est la notion du destinataire. Depuis quelque temps on se pose la question de savoir pour qui on traduit et dans quelle mesure on doit tenir compte de ce personnage parfois identifiable mais souvent anonyme qu'est le destinataire de la traduction.

Il y a au moins deux sortes de destinataires : le donneur d'ouvrage, normalement connu, et l'usager, qu'on ne connait personnellement que dans des cas exceptionnels. Le premier a commandé le travail et, payant la note, a le droit de le juger et de réclamer, le cas échéant. Le second a rarement l'occasion de donner son avis.

Le souci du destinataire est normal dans une société qui a le sens des relations publiques. Ajoutons que la traduction professionnelle est souvent un service commercial et que par conséquent le client entre en ligne de compte. Dans le cas du donneur d'ouvrage, le traducteur peut être amené à modifier sa traduction pour tenir compte d'une préférence ou d'un préjugé du client. Ainsi dans la traduction d'un bilan, il acceptera de remplacer intégré par consolidé. Dans le cas de l'usager, ce genre de rétroaction a peu de chance de se produire.

D'autre part, le traducteur peut avoir lui-même intérêt à savoir à qui il a affaire. Dans le cadre de la fonction publique, par exemple, il saura faire la différence entre un rapport impersonnel et le discours du ministre. Tout ceci pour dire qu'il y a en effet un problème du destinataire et que les traducteurs doivent se tenir en conséquence.

Plus généralement, on parle, on écrit pour être compris, et la transmissibilité d'une communication est un facteur important d'efficacité. Dans cette perspective, il vaut mieux parler de poêle que de cuisinière au Québec, et réserver ce dernier terme pour l'Europe francophone qui voit dans poêle uniquement un appareil de chauffage. De semblable façon, la question rituelle de la serveuse «Et comme breuvage?» sera insolite à Paris, alors qu'elle paraîtra naturelle à Québec.

Mais le souci du destinataire peut aller jusqu'à poser le problème de la responsabilité du traducteur. C'est là une question délicate, qu'il convient 
d'examiner posément. Il faut se garder d'étendre démesurément la responsabilité de qui que ce soit, sous peine de la diluer et de la ruiner. C'est un domaine dans lequel il ne faut pas courir trop de lièvres à la fois. On a dit que le traducteur a une triple obligation à l'égard de l'auteur, du message et du destinataire. Cette trilogie nous paraît excessive, bien qu'en fait elle puisse se réduire à deux termes, l'auteur ne faisant qu'un avec son message. De toute façon, elle a le tort de laisser de côté les deux points sur lesquels la responsabilité du traducteur est nettement engagée, à savoir le sens de l'original et le respect de la langue d'arrivée. Le traducteur a le devoir de restituer tout le sens et rien que le sens de l'original et il doit respecter le génie, ou la démarche, de la langue dans laquelle il traduit. C'est déjà beaucoup. Pour le reste on peut se demander si c'est bien de responsabilité qu'il s'agit et non pas plutôt de stratégies qui se recommandent par leur efficacité, à condition toutefois de ne pas amener le traducteur à transiger sur le sens de son texte ou sur la qualité de la langue qu'il emploie.

C'est un lieu commun de dire que la traduction a beaucoup évolué au cours des quarante dernières années. Non seulement elle a pris une grande importance dans une société avide de changement, mais elle est devenue plus complexe et comprend maintenant des sous-spécialités. Cela, tout le monde le sait. Ce qui nous a paru intéressant a été de montrer dans quelle mesure la réflexion théorique sur l'opération traduisante s'est infléchie et enrichie en subissant, à travers la pratique, le contrecoup des innovations qui se succèdent depuis le milieu du siècle. Nous avons naturellement souligné, chemin faisant, le côté positif de ces innovations, sans toutefois passer sous silence ce qui nous a paru discutable, et nous nous sommes alors permis une mise en garde. Comme on pouvait le prévoir, le bilan n'en est pas moins positif. 